\title{
Fingerprints of the conformal anomaly in the thermoelectric transport in Dirac and Weyl semimetals
}

\author{
Vicente Arjona, ${ }^{1, *}$ Maxim N. Chernodub, ${ }^{2,3, \dagger}$ and María A. H. Vozmediano ${ }^{1, \ddagger}$ \\ ${ }^{1}$ Instituto de Ciencia de Materiales de Madrid, C/Sor Juana Inés de la Cruz 3, Cantoblanco, 28049 Madrid, Spain \\ ${ }^{2}$ Institut Denis Poisson UMR 7013, Université de Tours, 37200 France \\ ${ }^{3}$ Laboratory of Physics of Living Matter, Far Eastern Federal University, Sukhanova 8, Vladivostok 690950, Russia
}

(Received 7 February 2019; published 10 June 2019)

\begin{abstract}
The conformal anomaly, a quantum anomaly related to metric deformations in conformally invariant systems, has been recently shown to give rise to a special contribution to the Nernst signal, which remains finite at zero temperature and chemical potential. In this work we provide a Kubo calculation that confirms the result of this unexpected signal in the conformal limit and extends the calculation to finite temperature and chemical potential. As a result, we predict a distinctive experimental signature of the conformal anomaly in the form of a plateau behavior in the thermoelectric coefficient as a function of the chemical potential in the extreme quantum limit.
\end{abstract}

DOI: 10.1103/PhysRevB.99.235123

\section{INTRODUCTION}

According to the Noether theorem, a continuum symmetry of a classical action gives rise to conserved currents and charges. Energy momentum and angular momentum are associated to space-time translation and rotations, respectively, while internal phase rotations of complex fields give rise to electric, color, or other conserved currents and charges. A quantum anomaly arises when a symmetry of the classical action cannot survive quantization. Normally, these anomalies occur in the presence of interactions and are caused by the necessity to introduce ultraviolet cutoffs when the classical currents are substituted by local operators $[1,2]$. The physical consequences of having quantum anomalies were first explored in the construction of quantum field theory (QFT) to describe elementary particles [1] and played an important role in grand unification and string theory. Nowadays, the interest on anomalies and anomaly-related transport has shifted to emergent condensed matter systems which support low-energy descriptions akin to their QFT partners $[3,4]$. In particular, Dirac and Weyl semimetals are 3D crystals having band crossings near the Fermi surface, whose low-energy quasiparticles are described by a massless Dirac Hamiltonian sharing all the properties of their high-energy partners. After an intense and successful analysis of the consequences of the chiral anomaly on magnetoelectric transport [5-8], the interest has shifted to gravitational effects, especially those of the mixed axial-gravitational anomaly $[9,10]$. These phenomena involve thermoelectric measurements in magnetic field.

The way in which gravity appears in material physics can be traced back to the problem of defining thermodynamic equilibrium in curved backgrounds [11], which culminated with the Luttinger theory of thermal transport [12]. The difficulty to find a local source for thermal (energy) currents

\footnotetext{
*vicente.arjona@csic.com

${ }^{\dagger}$ maxim.chernodub@gmail.com

†vozmediano@icmm.csic.es
}

was solved by introducing a (perhaps fictitious) gravitational field whose gradient plays the role of the electric field in the electromagnetic transport. This is a very natural choice in QFT where the stress-energy tensor is the response to variations of the metric: $T^{\mu \nu} \sim \delta S / \delta g_{\mu \nu}$.

Anomalies are generally due to vacuum fluctuations and often the induced transport responses persist at zero temperature and zero chemical potential. In a recent publication [13-15] a less-known quantum anomaly, the conformal anomaly (related to metric deformations), has been shown to give rise to a special contribution to the Nernst signal which remains finite at zero temperature and chemical potential.

Thermoelectric transport is a topic of major interest in technology and a very important tool to analyze the electronic properties of materials. From the early research it was known that semiconductors and semimetals are the best candidates to generate large figures of merit in thermopower, with bismuth, an almost compensated semimetal, holding the record for metallic compounds [16]. Dirac and Weyl semimetals belong naturally to the family of good thermoelectric materials and their thermoelectric properties are now at the center of interest in experimental and theoretical research [13,14,17-25]. Giant values of the anomalous Nernst effect are been systematically reported in the newly discovered magnetic Weyl semimetals. Most of the works deal with the anomalous Nernst and Hall effects (transverse transport in the absence of external applied magnetic fields) associated to the nontrivial Berry phase of the materials $[13,19,22,25,26]$. The corresponding anomalous coefficients are normally obtained using a semiclassical Boltzmann approach following the work in Ref. [27].

The regime of zero temperature and chemical potential, where the unusual prediction in [15] of a nonzero transport coefficient lies, prevents the use of the Boltzmann approach and the comparison with existing results. We present a Kubo calculation of the thermoelectric coefficient of the massless Dirac system in a magnetic field at zero chemical potential and zero temperature. The result coincides with the one obtained in Ref. [15] putting on firmer grounds the anomaly-related transport phenomena [3,4] in the Dirac matter. An extension 


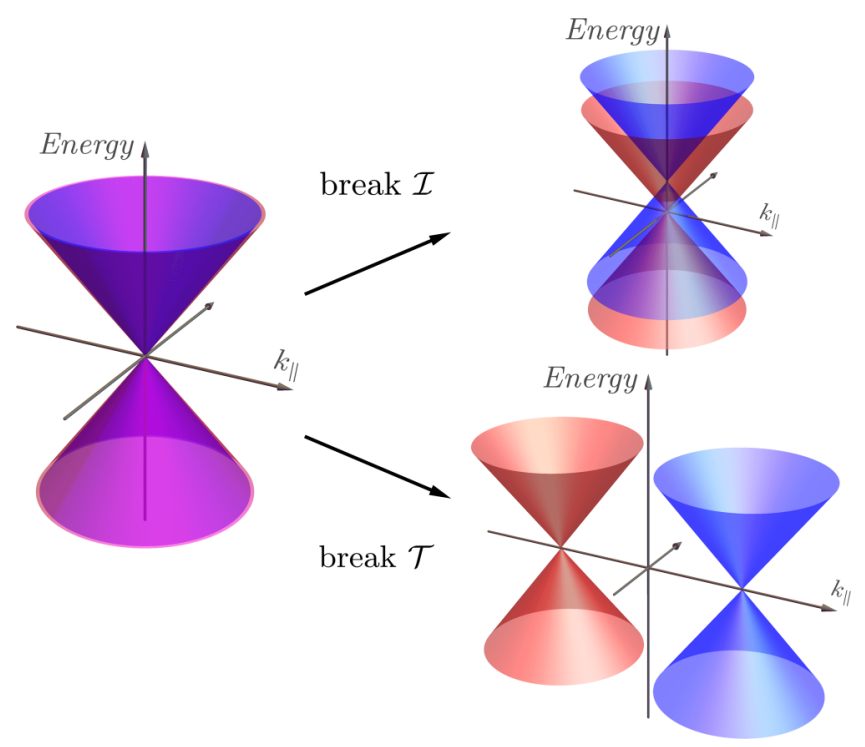

FIG. 1. Schematic dispersion relation of Dirac (left) and Weyl semimetals (right).

of the analysis to finite temperature and chemical potential confirms the robustness of the anomaly induced contribution and provides distinctive signatures for the experimental confirmation of this unexpected signal. The result is independent of the Berry curvature and hence it is common to Dirac and Weyl semimetals in the low- $T$ and low- $\mu$ limit.

\section{DIRAC AND WEYL SEMIMETALS}

The low-energy excitations around a nontrivial band crossing of a Dirac semimetal are described by the massless Dirac equation in three space dimensions. In the Weyl basis for the Dirac matrices, the Hamiltonian splits into two Weyl nodes (two-dimensional spinors) of definite chirality. The low-energy Hamiltonian around the Dirac point can be written as $H(k)=s \vec{\sigma} \cdot \vec{k}$, where $s= \pm$ is the chirality. Each chirality acts as a monopole of Berry curvature of charge $s$. In the material realizations, discrete symmetries associated to the crystal lattice plus standard inversion $\mathcal{I}$ and time reversal $\mathcal{T}$ play a crucial role. Depending of the symmetry of the crystal-and on the spin-orbit coupling-we find Dirac or Weyl semimetals schematically shown in Fig. 1. In the first class, the two chiralities are superimposed in momentum space and a mass term can arise mixing the two chiralities, unless the band crossing is protected by crystal symmetries. Examples of symmetry protected Dirac semimetals are $\mathrm{Cd}_{3} \mathrm{As}_{2}, \mathrm{Na}_{3} \mathrm{Bi}$ [28]. In these materials time-reversal symmetry is unbroken and the Hall conductivity is zero. In Weyl semimetals, the two chiralities are separated either in momentum or energy (see Fig. 1). This separation necessarily breaks either $\mathcal{T}$ or $\mathcal{I}$ and the Berry monopole makes the Weyl points very robust against perturbations. A gap in the spectrum can be opened only by merging the two chiralities.

Details of the material realizations can be found in many good reviews [28-30]. What is important for our work is to realize that the conformal invariance of the classical system implies that no dimension-full parameter enters into the description of the system. Our model will generically be that of a massless Dirac semimetal, as used in Ref. [15]. We will perform the calculation for each Weyl fermion and ensure that no cancellation occurs due to the contribution of opposite chiralities. Once this is confirmed, the result will equally apply to Weyl semimetals which, eventually, can receive additional contributions from the separation of the Weyl points (a dimension-full parameter).

\section{THERMOELECTRIC TRANSPORT: KUBO CALCULATION}

The response of an electronic system to a background electric field $\mathbf{E}$ and temperature gradient $\nabla T$ is parametrized as

$$
\begin{aligned}
& J^{i}=e^{2} L_{11}^{i j} E_{j}+e L_{12}^{i j} \nabla_{j} T \\
& J_{\epsilon}^{i}=e L_{21}^{i j} E_{j}+L_{22}^{i j} \nabla_{j} T,
\end{aligned}
$$

where the coefficients $L_{a b}^{i j}$ are related to the standard thermoelectric conductivities,

$$
J^{i}=\sigma^{i j} E_{j}+\alpha^{i j}\left(-\nabla_{j} T\right),
$$

by $\sigma^{i j}=e^{2} L_{11}^{i j}$ (electrical conductivity) and $\alpha^{i j}=-e L_{12}^{i j}$ (thermoelectric coefficient).

The current obtained in Ref. [15] for a single Dirac cone from the conformal anomaly (using the geometry $B_{z}, \nabla_{y} T$ ) was

$$
J^{x}=\frac{e^{2} v_{F} B}{18 \pi^{2} \hbar}\left(\frac{\nabla_{y} T}{T}\right),
$$

from where we extract the coefficient

$$
\chi^{x y}=\frac{e^{2} v_{F} B}{18 \pi^{2} \hbar} .
$$

This response is related with the standard definition in Eq. (1) by $\alpha^{i j}=\chi^{i j} / T$. In what follows we will present a standard Kubo formula calculation of this thermoelectric response. The calculation is straightforward but lengthy. We will sketch the main aspects and provide extensive information and details in Secs. III A and III B.

\section{A. Kubo formula for the thermoelectric tensor}

In linear response theory [31], when the action of a system is perturbed by a local source $F(t)$ which couples to an observable $B$ as $H_{F}(t)=F(t) B$, the change in the expectation value of any operator $A$ is assumed to be linear in the perturbing source: $\delta\left\langle A^{i}(t)\right\rangle=\int d t^{\prime} \chi^{i j}\left(t, t^{\prime}\right) F_{j}\left(t^{\prime}\right)$, and the response function $\chi^{i j}$ is given by the Kubo formula:

$$
\chi^{i j}\left(t, t^{\prime}\right)=-\frac{i}{\hbar} \int_{-\infty}^{\infty} d t^{\prime} \Theta\left(t-t^{\prime}\right)\left\langle\left[\hat{A}^{i}(t), \hat{B}^{j}\left(t^{\prime}\right)\right]\right\rangle_{0},
$$

where $\hat{A}$ is the the operator in the interaction picture representation and $\Theta(x)$ is the Heaviside function.

The problem of using a statistical variable (such as the temperature) as a (local) source coupling to an energy current was solved by Luttinger in Ref. [12]. Based on previous analyses by Tolman and Ehrenfest trying to define thermal equilibrium in a curved space [11], he proposed the gravitational potential 
$\Phi$ as the local source of thermal (energy) current $J_{\epsilon}^{i}$. Physically, the observation in Ref. [11] was that a temperature perturbation which moves a system out of equilibrium can be compensated by a variation in the gravitational potential such that, in equilibrium (we take the speed of light $c=1$ ),

$$
\nabla \Phi+\frac{\nabla T}{T}=0
$$

For small deviations from flat space the gravitational potential is proportional to the zero-zero component of the metric $\Phi \sim$ $g^{00}$, which couples to the energy density $T^{00}$. The perturbative Hamiltonian to be used in the linear response formalism is

$$
H_{\text {pert }}(t)=T^{00}(t) g_{00}(t),
$$

where it is assumed that the perturbation is switched on adiabatically. Using Eq. (5), the electric current generated by this perturbation is

$$
\begin{aligned}
\left\langle J^{i}\right\rangle(t, \mathbf{r})= & \int_{-\infty}^{\infty} d t^{\prime} d \mathbf{r}^{\prime}\left\{\frac{-i}{\hbar} \Theta\left(t-t^{\prime}\right)\left\langle\left[J^{i}(t, \mathbf{r}), T^{00}\left(t^{\prime}, \mathbf{r}^{\prime}\right)\right]\right\rangle\right\} \\
& \times g_{00}\left(t^{\prime}, \mathbf{r}^{\prime}\right) .
\end{aligned}
$$

To get the response to the spacial derivative of $g^{00}$, we use the conservation law of the energy-momentum tensor $T^{\mu \nu}$,

$$
\partial_{0} T^{00}(t, \mathbf{r})+v_{\mathrm{F}} \partial_{i} T^{0 i}(t, \mathbf{r})=0,
$$

where we have introduced the Fermi velocity in the spatial part of the metric to adapt the calculation to the case of Dirac semimetals. The zero-zero component of the energymomentum tensor is then:

$$
T^{00}(t, \mathbf{r})=-\int_{-\infty}^{t} d t^{\prime} v_{F} \partial_{i} T^{0 i}\left(t^{\prime}, \mathbf{r}\right),
$$

where we have used that the system is unperturbed at $t=$ $-\infty$. Introducing Eq. (10) in Eq. (8) and integrating by parts, we get:

$$
\begin{aligned}
\left\langle J^{i}\right\rangle(t, \mathbf{r})= & \int_{-\infty}^{\infty} d t^{\prime} d \mathbf{r}^{\prime} \int_{-\infty}^{t^{\prime}} d t^{\prime \prime}\left\{\frac{-i v_{F}}{\hbar} \Theta\left(t-t^{\prime}\right)\right. \\
& \left.\times\left\langle\left[J^{i}(t, \mathbf{r}), T^{0 j}\left(t^{\prime \prime}, \mathbf{r}^{\prime}\right)\right]\right\rangle\right\} \partial_{j} g_{00}\left(t^{\prime}, \mathbf{r}^{\prime}\right) .
\end{aligned}
$$

Equation (11) represents the electric current generated by a thermal gradient computed via Kubo formula. The Fourier transform

$$
\left\langle J^{i}\right\rangle(\omega, \mathbf{q})=\chi^{i j}(\omega, \mathbf{q})\left(i q_{j}\right) g_{00}(\omega, \mathbf{q}),
$$

leads to the standard form of the response function:

$$
\begin{aligned}
\chi^{i j}(\omega, \mathbf{q})= & (2 \pi)^{3} \int d t e^{i \omega\left(t-t^{\prime}\right)} \int_{-\infty}^{t^{\prime}} d t^{\prime \prime}\left\{\frac{-i v_{F}}{\mathcal{V} \hbar} \Theta\left(t-t^{\prime}\right)\right. \\
& \left.\left\langle\left[J^{i}(t, \mathbf{q}), T^{0 j}\left(t^{\prime \prime},-\mathbf{q}\right)\right]\right\rangle\right\}
\end{aligned}
$$

where we have assumed that the system is invariant under time and spatial translations and $\mathcal{V}$ is the volume of the sample.
Equation (13) is the expression that we will compute for a Dirac semimetal in the presence of an external magnetic field.

It is important to note that, in the Luttinger approach described in this section, the current computed with the Kubo formula (11) includes local magnetization currents not contributing to transport [32,33]. The transport coefficient in (1) will be given by $L_{12}^{i j}=\chi_{12}^{i j}-\epsilon^{i j l} M_{l}$ [21], where $M_{l}$ is the magnetization [34]. Our main result Eq. (24) in the local limit at zero temperature and chemical potential is not affected by this term.

\section{B. Highlights of the calculation}

We have done the calculation using the Lehman representation of the Green's function as described in Ref. [31]. The Hamiltonian of a Dirac semimetal in an external magnetic field can be decomposed into two Weyl Hamiltonians,

$$
H_{s}=s v_{F} \sigma^{i}\left(p_{i}+e A_{i}\right)
$$

where $s$ describes the chirality of the node, $p_{i}$ is the momentum operator, and $e$ is the charge of the electron $(e=|e|)$. Choosing the magnetic field in the $z$ direction and using the Landau gauge $A_{x}=-B y$, the spectrum of the Hamiltonian is

$$
\begin{aligned}
E_{k_{z} m s} & =\operatorname{sign}(m) v_{F}\left[2 e \hbar B|m|+\hbar^{2} k_{z}^{2}\right]^{1 / 2} \quad m \in \mathbb{Z}, m \neq 0, \\
E_{k_{z} 0 s} & =-s v_{F} \hbar k_{z} .
\end{aligned}
$$

The two zeroth Landau levels have opposite chiralities and the rest are doubly degenerated. The eigenvectors are

$$
\begin{aligned}
\varphi_{\mathbf{k} m s}(\mathbf{r})= & \frac{1}{\sqrt{L_{x} L_{z}}} \frac{e^{i k_{x} x} e^{i k_{z} z}}{\sqrt{\alpha_{k_{z}, m, s}^{2}+1}} e^{-\left(y-k_{x} l_{B}^{2}\right)^{2} / 2 l_{B}^{2}} \\
& \times\left(\begin{array}{c}
\frac{\alpha_{k_{z} m s}}{\sqrt{2^{M-1}(M-1) ! \pi^{1 / 2} l_{B}}} H_{M-1}\left[\frac{y-k_{x} l_{B}^{2}}{l_{B}}\right] \\
\frac{1}{\sqrt{2^{M} M ! \pi^{1 / 2} l_{B}}} H_{M}\left[\frac{y-k_{x} l_{B}^{2}}{l_{B}}\right]
\end{array}\right),
\end{aligned}
$$

with

$$
\alpha_{k_{z}, m, s}=\frac{-\sqrt{2 e B \hbar|m|}}{E_{k_{z} m s} / s v_{F}-\hbar k_{z}} .
$$

Capital letters refer to the absolute value of Landau levels, $H_{m}(x)$ are the Hermite polynomials, and the factor $\left(\alpha_{k_{z}, m, s}^{2}+\right.$ $1)^{1 / 2}$ comes from the wave-function normalization. In the basis of the Landau levels, the current operators in Eq. (13) read:

$$
\begin{gathered}
\hat{J}^{x}(t, \mathbf{q})=\sum_{\mathbf{k}, m n} J_{\mathbf{k} m s, \mathbf{k}+\mathbf{q} n s}^{x}(\mathbf{q}) \hat{a}_{\mathbf{k} m s}^{\dagger}(t) \hat{a}_{\mathbf{k}+\mathbf{q} n s}(t), \\
\hat{T}^{0 y}\left(t^{\prime \prime},-\mathbf{q}\right)=\sum_{\boldsymbol{\kappa}, \mu \nu} T_{\kappa \mu s, \boldsymbol{\kappa}-\mathbf{q} v s}^{0 y}(\mathbf{q}) \hat{a}_{\boldsymbol{\kappa} \mu s}^{\dagger}\left(t^{\prime \prime}\right) \hat{a}_{\boldsymbol{\kappa}-\mathbf{q} \nu s}\left(t^{\prime \prime}\right),
\end{gathered}
$$

where the matrix elements (for a detailed derivation, see Sec. III A) are

$$
J_{\mathbf{k} m s, \mathbf{k}+\mathbf{q} n s}^{x}(\mathbf{q})=\frac{1}{(2 \pi)^{3 / 2}} \int d y e^{-i q_{y} y} \operatorname{sv}_{F} e \varphi_{\mathbf{k} m s}^{*}(y) \sigma^{x} \varphi_{\mathbf{k}+\mathbf{q} n s}(y),
$$




$$
\begin{aligned}
T_{\kappa \mu s, \kappa-\mathbf{q} \nu s}^{0 y}(\mathbf{q})= & \frac{1}{4} \frac{1}{(2 \pi)^{3 / 2}} \int d y e^{+i q_{y} y}\left[v_{F} \varphi_{\kappa \mu s}^{*}(y) \mathbb{I} p_{y} \varphi_{\kappa-\mathbf{q} \nu s}(y)-v_{F} p_{y} \varphi_{\kappa \mu s}^{*}(y) \amalg \varphi_{\kappa-\mathbf{q} \nu s}(y)\right] \\
& +\frac{1}{4} \frac{1}{(2 \pi)^{3 / 2}} \int d y e^{+i q_{y} y} \varphi_{\kappa \mu s}^{*}(y) s \sigma^{y}\left(E_{\kappa \mu s}+E_{\kappa-\mathbf{q} \nu s}-2 \mu\right) \varphi_{\kappa-\mathbf{q} \nu s}(y) .
\end{aligned}
$$

Introducing Eqs. (18)-(21) into Eq. (13) the response function is given by:

$$
\chi^{x y}(\omega, \mathbf{q})=\lim _{\eta \rightarrow 0^{+}} \sum_{\mathbf{k}, m n} \frac{(2 \pi)^{3}}{\mathcal{V}} \frac{i v_{F} \hbar J_{\mathbf{k} m s, \mathbf{k}+\mathbf{q} n s}^{x}(\mathbf{q}) T_{\mathbf{k}+\mathbf{q} n s, \mathbf{k} m s}^{0 y}(\mathbf{q})}{\left(E_{k_{z} m s}-E_{k_{z}+q_{z} n s}+i \hbar \eta\right)\left(E_{k_{z} m s}-E_{k_{z}+q_{z} n s}+\hbar \omega+i \hbar \eta\right)}\left[n_{\mathbf{k} m s}-n_{\mathbf{k}+\mathbf{q} n s}\right],
$$

where we have used the relation

$$
\left\langle\left[\hat{a}_{\mathbf{k} m s}^{\dagger} \hat{a}_{\mathbf{k}+\mathbf{q} n s}, \hat{a}_{\kappa \mu s}^{\dagger} \hat{a}_{\kappa-\mathbf{q} v s}\right]\right\rangle=\delta_{\mathbf{k}, \kappa-\mathbf{q}} \delta_{m, \nu} \delta_{\mathbf{k}+\mathbf{q}, \kappa} \delta_{n, \mu}\left\{n_{\mathbf{k} m s}-n_{\mathbf{k}+\mathbf{q} n s}\right\}
$$

and we have introduced a factor $e^{+\eta\left(t^{\prime \prime}-t\right)}$ in the time integration to guarantee the convergence.

As we see, there is a sum over energetically allowed transitions. In the limit $T \rightarrow 0$, the distribution function becomes a step function: $n_{\mathbf{k} m s}=\Theta\left(\mu-E_{k_{z} m s}\right)$ and only transitions between positive and negative levels are allowed (when the chemical potential is placed at the neutrality point). We have evaluated the numerical value of the response function by considering only the dominant contribution due to transitions between the lowest Landau levels $(0, \pm 1)$. The final result is

$$
\chi^{x y} \equiv \lim _{\omega \rightarrow 0} \lim _{\mathbf{q} \rightarrow 0} \chi^{x y}(\omega, \mathbf{q})=\frac{1}{2(2 \pi)^{2}} \frac{v_{F} e^{2} B}{\hbar} .
$$

The contribution to the numerical coefficient coming from higher-energy transitions decrease very rapidly with the Landau level $N$. Summing up the contributions up to $N=20$ changes the numerical value by a factor of 2 approximately. Finite temperature and chemical potential dependencies will be discussed in the next section.

\section{EXPERIMENTAL SIGNATURES. PROSPECTS}

The calculation presented here is based on a threedimensional massless Dirac Hamiltonian that can describe Dirac or Weyl semimetals in the low-energy regime. The result is valid irrespective of whether the opposite chiralities are superimposed (Dirac) or separated (Weyl) in momentum space. Additional contributions proportional to the separation of the Weyl nodes may arise in Weyl semimetals. We have been particularly careful to follow the chirality dependence of all the terms along the calculation to ensure that no cancellations occur (both chiralities contribute to the response function with the same sign). Placing the chemical potential at $\mu=0$ and adding the contributions of the two chiralities, the thermoelectric coefficient $\alpha^{x y}$ in Eq. (1) is given by:

$$
\alpha^{x y}=\frac{2}{T} \chi^{x y}=\frac{e^{2} v_{F} B}{4 \pi^{2} T \hbar},
$$

which coincides with the result in Ref. [15] up to a numerical factor close to unity (the sign is a matter of convention). This is a remarkable result. First notice that, although we deal with thermally induced transport, the calculation has been done at zero temperature and the coefficient remains finite in the limit $T \rightarrow 0$, with $\nabla T / T$ being kept finite. Second, this calculation is valid at the Dirac point, at zero chemical potential, where it captures the vacuum contribution from the quantum conformal anomaly. In real materials, the Fermi energy is close but not exactly at the Dirac point. To approach the experimental situation, we have extended the calculation to include a finite chemical potential $\mu$ and temperature. Figure 2 shows the Landau-level spectrum around a single Weyl node of the Dirac semimetal in normalized units. $\omega_{c}$ is the cyclotron frequency. The straight line corresponds to the chiral zeroth Landau level. The horizontal line in the inset is the numerical result obtained for the response function $\chi^{x y}$ when the chemical potential lies in the interval $|\mu|<\hbar \omega_{c}$. This is a consistency check since the zeroth Landau level has a constant density of states. The contribution from the opposite chirality has the same sign. In Fig. 3(a) we plot the value of the thermoelectric coefficient as a function of the chemical potential in the same range $|\mu|<\hbar \omega_{c}$ for different values of the temperature up to $k_{B} T=0.1 \hbar \omega_{c}$. Thermally activated carriers contribute to higher values of the transport coefficient and the size of the plateau (constant value around $\mu=0$ ) is reduced according to the Fermi Dirac distribution. Figure 3(b) shows the behavior of the thermoelectric coefficient as a

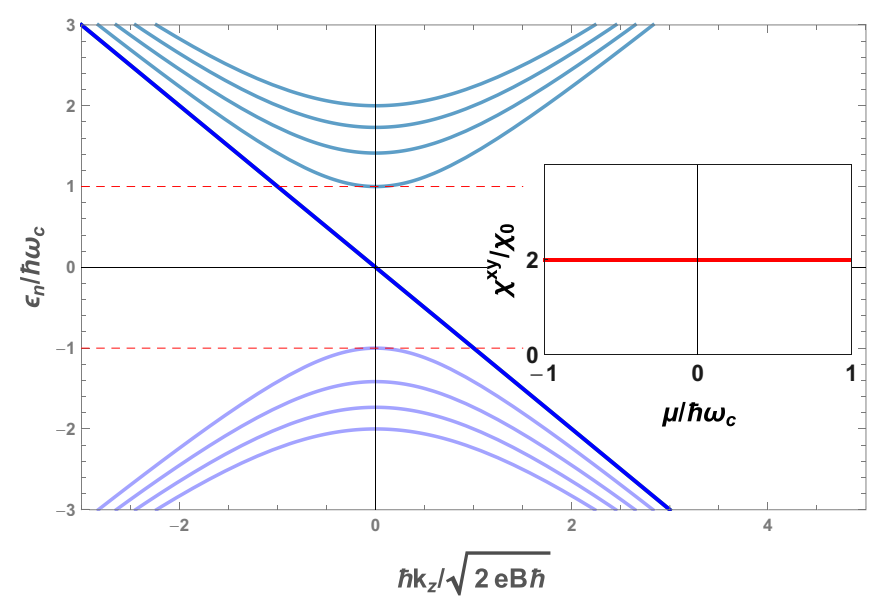

FIG. 2. Landau-level structure of a single chirality in the Dirac semimetal. The inset shows the calculated thermoelectric coefficient as a function of the chemical potential (in normalized units) at $T=0$. The function has a constant value $\left[\chi^{x y} / \chi_{0}=2\right.$, where $\chi_{0}=$ $\left.v_{F} e^{2} B / 4(2 \pi)^{2} \hbar\right]$ when $\mu$ lies in the interval between the first Landau levels $n= \pm 1$. The opposite chirality contributes to the transport with the same sign. 

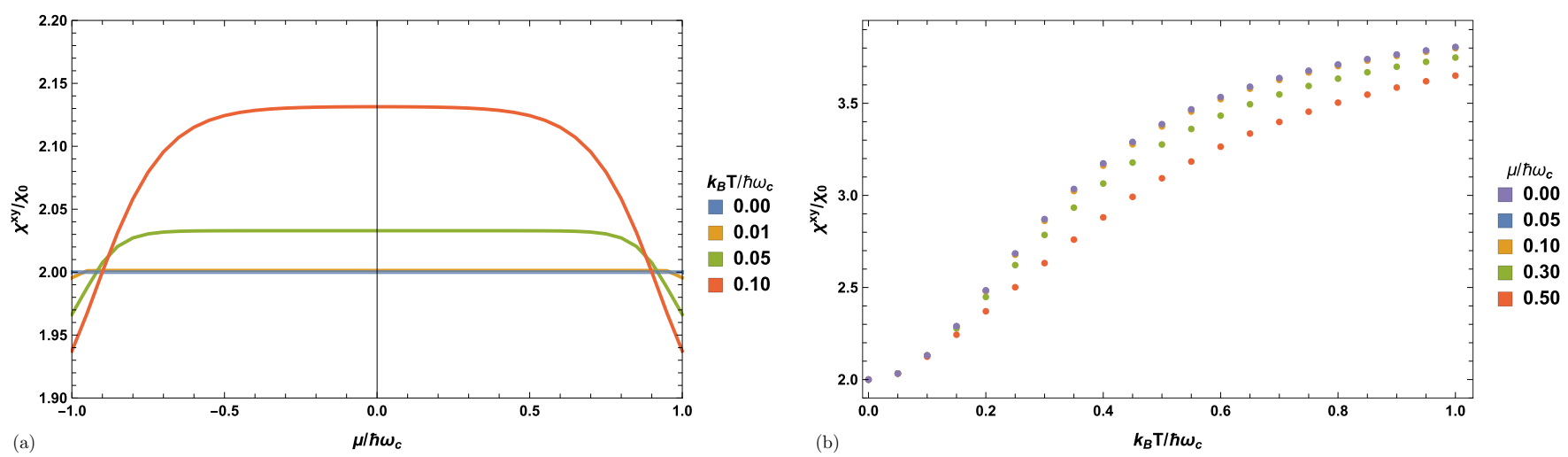

FIG. 3. (a) Behavior of the thermoelectric coefficient as a function of the chemical potential $\mu$ for fixed values of $k_{B} T / \hbar \omega_{c}$. Thermally excited carriers enhance the transport and the boundaries of the plateau are smoothen by the thermal distribution function. (b) Behavior of the thermoelectric coefficient as a function of the temperature for different values of $\mu$ in the interval $|\mu|<\hbar \omega_{c}$.

function of the temperature for different values of $\mu$ in the interval $|\mu|<\hbar \omega_{c}$.

The best materials to explore the physics described in this work would be Dirac or Weyl semimetals with the Fermi level as close as possible to the Dirac point. As discussed in the text, the calculation has been done for a single chirality and we have checked that the contribution from the other chirality adds up. So the result is valid irrespective of the relative positions of the Weyl cones. The advantage of Dirac semimetals, where the two chiralities are superimposed in momentum and energy, is that it minimizes the anomalous contribution coming from the Berry phase (although there will be an anomalous contribution in the presence of a magnetic field) [13]. In the case of Weyl semimetals with the Weyl nodes separated in momentum space, there will be an additional contribution proportional to the separation of the Weyl nodes. Since this parameter breaks conformal invariance explicitly we have not considered it in this work. As mentioned in Ref. [15], we can also measure the conformal anomaly contribution to the Nernst effect indirectly in these materials by measuring the $S_{x x}$ component of the thermopower. An interesting material to explore the conformal anomaly contribution to thermal transport would be GdPtBi, which has the Fermi level near the Dirac point and has recently been used to discuss the mixed gravitational anomaly contribution [10].

We must note that the Kubo formula does not always provide the thermoelectric coefficients correctly. In the presence of nonzero gradients of the chemical potential and/or temperature the Kubo formalism includes magnetization currents not contributing to the transport $[32,33]$. The energy magnetization current affects the thermal conductivity but does not alter the thermoelectric coefficients. Moreover, at zero chemical potential and zero temperature the magnetization current vanishes.

In the physics of massless Dirac materials, an anomalous nonconservation of a current is often the price to pay for another current to be conserved. In the case of the chiral anomaly, we chose to conserve the vector current (and hence the electric charge) and the axial charge to be anomalous. A similar scenario occurs with the conformal anomaly. The Noether current associated to the scale invariance, the dilatation current, is $J_{D}^{\mu}=T^{\mu v} x_{v}$. Assuming energy-momentum conservation, its divergence is then the trace of the energymomentum tensor. We could have chosen to conserve the dilatation current at the quantum level at the price of breaking energy-momentum conservation. Part of the relevance of the material realization of Weyl physics is the possibility to explore these choices and get a deeper insight into the physics of anomalies.

Note added. After the submission of this manuscript, two experimental results have appeared where a plateau in the thermopower similar to the one described in this work is observed in the Dirac semimetal $\mathrm{ZrTe}_{5}$ [35] and in the Weyl semimetal TaP [36]. See also Ref. [37].

\section{ACKNOWLEDGMENTS}

We thank A. Cortijo for invaluable help with the calculations and discussion. Useful comments from Y. Ferreiros, F. de Juan, P. Kim, K. Landsteiner, and I. Shovkovy are also acknowledged. This work has been supported by the PIC2016FR6/PICS07480, Spanish MECD Grant No. FIS2014-57432-P, European Union structural funds and the Comunidad Autónoma de Madrid NMAT2D-CM Program No. S2018/NMT-4511, and within the state assignment of the Ministry of Science and Higher Education of Russia (Grant No. 3.6261.2017/8.9).

\section{APPENDIX A: PROJECTION OF THE OPERATORS ON THE LANDAU WAVE FUNCTIONS}

The Hamiltonian of the Dirac semimetal in a magnetic field is decomposed into the following two Weyl Hamiltonians:

$$
H_{s}=s v_{F} \sigma^{i}\left(p_{i}+e A_{i}\right)
$$

where $s= \pm$ denotes the chirality of the node. In the Landau gauge $A_{x}=-B y$, the spectrum of the system is

$$
\begin{gathered}
E_{k_{z} m s}=\operatorname{sign}(m) v_{F}\left[2 e \hbar B|m|+\hbar^{2} k_{z}^{2}\right]^{1 / 2} \quad m \in \mathbb{Z}, m \neq 0 \\
E_{k_{z} 0 s}=-s v_{F} \hbar k_{z},
\end{gathered}
$$


and the eigenvectors are

$$
\varphi_{\mathbf{k} m s}(\mathbf{r})=\frac{1}{\sqrt{L_{x} L_{z}}} \frac{e^{i k_{x} x} e^{i k_{z} z}}{\sqrt{\alpha_{k_{z} m s}^{2}+1}} e^{-\left(y-k_{x} l_{B}^{2}\right)^{2} / 2 l_{B}^{2}}\left(\begin{array}{c}
\frac{\alpha_{k_{z} m s}}{\sqrt{2^{M-1}(M-1) ! \pi^{1 / 2} l_{B}}} H_{M-1}\left[\frac{y-k_{x} l_{B}^{2}}{l_{B}}\right] \\
\frac{1}{\sqrt{2^{M} M ! \pi^{1 / 2} l_{B}}} H_{M}\left[\frac{y-k_{x} l_{B}^{2}}{l_{B}}\right]
\end{array}\right) .
$$

The term $\alpha_{k_{z} m s}$ is chiral dependent only when the Landau level $m$ is different from 0 . The field operators are expressed, in the Landau basis, as:

$$
\begin{gathered}
\hat{\psi}^{\dagger}(t, \mathbf{r})=\sum_{\mathbf{k} m}\langle\mathbf{k} m s \mid \mathbf{r}\rangle \hat{a}_{\mathbf{k} m s}^{\dagger}(t)=\sum_{\mathbf{k} m} \varphi_{\mathbf{k} m s}^{*}(\mathbf{r}) \hat{a}_{\mathbf{k} m s}^{\dagger}(t), \\
\hat{\psi}(t, \mathbf{r})=\sum_{\mathbf{l} n}\langle\mathbf{r} \mid \mathbf{l} n s\rangle \hat{a}_{\mathbf{l} n s}(t)=\sum_{\mathbf{l} n} \varphi_{\mathbf{l} n s}(\mathbf{r}) \hat{a}_{\mathbf{l} n s}(t) .
\end{gathered}
$$

Using Eqs. (A5) and (A6), the current operator and the energy-momentum tensor defined in Eq. (13) are trivially represented in the Landau basis:

$$
\begin{aligned}
J^{x}(t, \mathbf{r})=s v_{F} e \sum_{\mathbf{k} m, \mathbf{l} n} \varphi_{\mathbf{k} m s}^{*}(\mathbf{r}) \sigma^{x} \varphi_{\mathbf{l} n s}(\mathbf{r}) \hat{a}_{\mathbf{k} m s}^{\dagger}(t) \hat{a}_{\mathbf{l n s}}(t), \\
T^{0 y}\left(t^{\prime \prime}, \mathbf{r}^{\prime}\right)=\sum_{\kappa \mu, \lambda \nu} \frac{1}{4}\left[v_{F} \varphi_{\kappa \mu s}^{*}\left(\mathbf{r}^{\prime}\right) \mathbb{I}\left\{p_{y} \varphi_{\lambda \nu s}\left(\mathbf{r}^{\prime}\right)\right\}-v_{F}\left\{p_{y} \varphi_{\kappa \mu s}^{*}\left(\mathbf{r}^{\prime}\right)\right\} \mathbb{I} \varphi_{\lambda \nu s}\left(\mathbf{r}^{\prime}\right)\right] \hat{a}_{\kappa \mu s}^{\dagger}\left(t^{\prime \prime}\right) \hat{a}_{\lambda v s}\left(t^{\prime \prime}\right) \\
+\sum_{\kappa \mu, \lambda v} \frac{1}{4}\left[\varphi_{\kappa \mu s}^{*}\left(\mathbf{r}^{\prime}\right) s \sigma^{y} \varphi_{\lambda \nu s}\left(\mathbf{r}^{\prime}\right)\right]\left[\hat{a}_{\kappa \mu s}^{\dagger}\left(t^{\prime \prime}\right)\left\{i \hbar \partial_{0} \hat{a}_{\lambda v s}\left(t^{\prime \prime}\right)\right\}-\left\{i \hbar \partial_{0} \hat{a}_{\kappa \mu s}^{\dagger}\left(t^{\prime \prime}\right)\right\} \hat{a}_{\lambda v s}\left(t^{\prime \prime}\right)\right] \\
+\sum_{\kappa \mu, \lambda v} \frac{1}{4}\left[\varphi_{\kappa \mu s}^{*}\left(\mathbf{r}^{\prime}\right) s \sigma^{y}(2 \mu) \varphi_{\lambda v s}\left(\mathbf{r}^{\prime}\right)\right] \hat{a}_{\kappa \mu s}^{\dagger}\left(t^{\prime \prime}\right) \hat{a}_{\lambda v s}\left(t^{\prime \prime}\right),
\end{aligned}
$$

where, for future purposes, we have introduced a finite chemical potential in the last term of the energy-momentum tensor. All time dependence is given explicitly by the second quantization operators: $\hat{a}_{\mathbf{k} m s}^{\dagger}(t)=e^{i / \hbar E_{k_{z} m s} t} \hat{a}_{\mathbf{k} m s}^{\dagger}$. In momentum space, we get:

$$
\begin{gathered}
\hat{J}^{x}(t, \mathbf{q})=\sum_{\mathbf{k} m, \mathbf{l} n} J_{\mathbf{k} m s, \mathbf{l n} n s}^{x}(\mathbf{q}) \hat{a}_{\mathbf{k} m s}^{\dagger}(t) \hat{a}_{\mathbf{l} n s}(t), \\
\hat{T}^{0 y}\left(t^{\prime \prime},-\mathbf{q}\right)=\sum_{\kappa \mu, \lambda v} T_{\kappa \mu s, \lambda v s}^{0 y}(\mathbf{q}) \hat{a}_{\kappa \mu s}^{\dagger}\left(t^{\prime \prime}\right) \hat{a}_{\lambda \nu s}\left(t^{\prime \prime}\right),
\end{gathered}
$$

where the matrix elements are written as a function of the Landau eigenvectors:

$$
\begin{aligned}
& J_{\mathbf{k} m s, \mathbf{l} n s}^{x}(\mathbf{q})=\frac{1}{(2 \pi)^{3 / 2}} \int d \mathbf{r} e^{-i \mathbf{q r}} s v_{F} e \varphi_{\mathbf{k} m s}^{*}(\mathbf{r}) \sigma^{x} \varphi_{\mathbf{l} n s}(\mathbf{r}), \\
& T_{\kappa \mu s, \lambda v s}^{0 y}(\mathbf{q})=\frac{1}{4} \frac{1}{(2 \pi)^{3 / 2}} \int d \mathbf{r} e^{+i \mathbf{q r}}\left[v_{F} \varphi_{\kappa \mu s}^{*}(\mathbf{r}) \mathbb{I}\left\{p_{y} \varphi_{\lambda \nu s}(\mathbf{r})\right\}-v_{F}\left\{p_{y} \varphi_{\kappa \mu s}^{*}(\mathbf{r})\right\} \mathbb{I} \varphi_{\lambda \nu s}(\mathbf{r})\right] \\
& +\frac{1}{4} \frac{1}{(2 \pi)^{3 / 2}} \int d \mathbf{r} e^{+i \mathbf{q r}} \varphi_{\kappa \mu s}^{*}(\mathbf{r}) s \sigma^{y}\left(E_{\kappa_{z} \mu s}+E_{\lambda_{z} \nu s}-2 \mu\right) \varphi_{\lambda \nu s}(\mathbf{r}) .
\end{aligned}
$$

In the chosen gauge, $k_{x}$ and $k_{z}$ are still good quantum numbers, and their wave functions are plane waves [see Eq. (A4)]. This allows us to establish a relation between the wave vectors $\mathbf{k}, \mathbf{l}, \boldsymbol{\kappa}$, and $\lambda$. Considering the occupied volume per value, we can replace the summation over $\mathbf{l}$ and $\lambda$ in Eqs. (A9) and (A10) by integrals:

$$
\sum_{\mathbf{l}}=\frac{L_{x} L_{y}}{4 \pi^{2}} \int d l_{x} d l_{z}
$$

and get two Dirac $\delta$ functions correlating the wave vectors:

$$
\begin{aligned}
& J_{\mathbf{k} m s, \mathbf{l n s}}^{x}(\mathbf{q})=\frac{1}{(2 \pi)^{3 / 2}} \int d l_{x} d l_{z} \int d y e^{-i q_{y} y} \delta\left(l_{x}-k_{x}-q_{x}\right) \delta\left(l_{z}-k_{z}-q_{z}\right) s v_{F} e \varphi_{\mathbf{k} m s}^{*}(y) \sigma^{x} \varphi_{\mathbf{l} n s}(y), \\
& T_{\kappa \mu s, \lambda \nu s}^{0 y}(\mathbf{q})=\frac{1}{(2 \pi)^{3 / 2}} \int d \lambda_{x} d \lambda_{z} \int d y e^{i q_{y} y} \delta\left(\lambda_{x}-\kappa_{x}+q_{x}\right) \delta\left(\lambda_{z}-\kappa_{z}+q_{z}\right) \varphi_{\kappa \mu s}^{*}(y)(\cdots) \varphi_{\lambda \nu s}(y),
\end{aligned}
$$


where $\varphi_{\mathbf{k} m s}(y)$ is the remaining part of the wave function that only depends on $y$ :

$$
\varphi_{\mathbf{k} m s}(y)=\frac{1}{\sqrt{\alpha_{k_{z} m s}^{2}+1}} e^{-\left(y-k_{x} l_{B}^{2}\right)^{2} / 2 l_{B}^{2}}\left(\begin{array}{c}
\frac{\alpha_{k_{z}, m, s}}{\sqrt{2^{M-1}(M-1) ! \pi^{1 / 2} l_{B}}} H_{M-1}\left[\frac{y-k_{x} l_{B}^{2}}{l_{B}}\right] \\
\frac{1}{\sqrt{2^{M} M ! \pi^{1 / 2} l_{B}}} H_{M}\left[\frac{y-k_{x} l_{B}^{2}}{l_{B}}\right]
\end{array}\right) .
$$

Performing the different integrals one obtains the expressions given in Eqs. (20) and (21) of the main text.

\section{APPENDIX B: THERMOELECTRIC RESPONSE}

The thermoelectric current generated by an external thermal gradient perpendicular to a magnetic field in a Dirac or Weyl semimetal is described, in momentum space, by:

$$
\left\langle J^{x}\right\rangle(\omega, \mathbf{q})=\chi^{x y}(\omega, \mathbf{q})\left(i q_{y}\right) g_{00}(\omega, \mathbf{q})
$$

where the response function is written as:

$$
\chi^{x y}(\omega, \mathbf{q})=\lim _{\eta \rightarrow 0} \sum_{\mathbf{k}, m n} \frac{(2 \pi)^{3}}{\mathcal{V}} \frac{i v_{F} \hbar J_{\mathbf{k} m s, \mathbf{k}+\mathbf{q} n s}^{x}(\mathbf{q}) T_{\mathbf{k}+\mathbf{q} n s, \mathbf{k} m s}^{0 y}(\mathbf{q})}{\left(E_{k_{z} m s}-E_{k_{z}+q_{z} n s}+i \hbar \eta\right)\left(E_{k_{z} m s}-E_{k_{z}+q_{z} n s}+\hbar \omega+i \hbar \eta\right)}\left[n_{\mathbf{k} m s}-n_{\mathbf{k}+\mathbf{q} n s}\right] .
$$

The rest of the calculation reduces to computing the numerical value of Eq. (B2). The integration over $k_{x}$ is easily done since the Landau levels do not depend on it; only the eigenvectors are proportional to this conserved number. It can be shown that the product of matrix elements does not depend on $k_{x}$ either; it is a function of the wave vector $\mathbf{q}$ only. Consequently, the integration over $k_{x}$ simplifies gives only the degeneracy factor $e B L_{y} / \hbar$.

In order to facilitate the integration over $k_{z}$, we define a dimensionless variable $\kappa_{z}=\hbar k_{z} / \sqrt{2 e B \hbar}$. The eigenvalues are rewritten in the form $E_{k_{z} m s}=v_{F}(2 e B \hbar)^{1 / 2} \mathcal{E}_{\kappa_{z} m s}$, with $\mathcal{E}_{k_{z} m s}$ a dimensionless energy whose expression depends on the given level. The parameter $\alpha_{k_{z} m s}$ is also normalized with this change.

\section{Energy-momentum tensor: Product of Hermite polynomials}

We will split the matrix elements of the energy-momentum tensor, defined in Eqs. (A12) and (A15), into three parts:

$$
\begin{gathered}
T_{\mathbf{k}+\mathbf{q} n s, \mathbf{k} m s}^{0 y[1]}(\mathbf{q})=\frac{1}{4(2 \pi)^{3 / 2}} \int d y e^{+i q_{y} y} \varphi_{\mathbf{k}+\mathbf{q} n s}^{*}(y) s \sigma^{y}\left(E_{\kappa_{z} \mu s}+E_{\lambda_{z} v s}-2 \mu\right) \varphi_{\mathbf{k} m s}(y), \\
T_{\mathbf{k}+\mathbf{q} n s, \mathbf{k} m s}^{0 y[2]}(\mathbf{q})=\frac{1}{4(2 \pi)^{3 / 2}} \int d y e^{+i q_{y} y} v_{F} \varphi_{\mathbf{k}+\mathbf{q} n s}^{*}(y) \mathbb{I}\left\{p_{y} \varphi_{\mathbf{k} m s}(y)\right\}, \\
T_{\mathbf{k}+\mathbf{q} n s, \mathbf{k} m s}^{0 y[3]}(\mathbf{q})=\frac{-1}{4(2 \pi)^{3 / 2}} \int d y e^{+i q_{y} y} v_{F}\left\{p_{y} \varphi_{\mathbf{k}+\mathbf{q} n s}^{*}(y)\right\} \Pi \varphi_{\mathbf{k} m s}(y) .
\end{gathered}
$$

It is important to notice that only the first expression Eq. (B3) depends on the chirality of the node. The operator $p_{y}$ in Eqs. (B4) and (B5) produces two results when acting on the wave functions; the first one comes from the exponential factor $\exp \left[-\left(y-k_{x} l_{B}^{2}\right)^{2} / l_{B}^{2}\right]$ inside $\varphi_{\mathbf{k} m s}$. When adding the two contributions from Eqs. (B4) an (B5), a term proportional to $q_{x}$ is produced. This term vanishes when the local limit is considered. The second term comes from the derivative acting on the Hermite polynomials, giving two different terms. Those three contributions will be multiplied by the current operator to provide the thermoelectric response.

When computing the expressions of the matrix elements Eqs. (A11) and (A12), each current operator is given by the product of Hermite polynomials that satisfy the formula [38]:

$$
\int_{-\infty}^{\infty} d y e^{-y^{2}} H_{r}(y+a) H_{s}(x+b)=2^{s} r ! \pi^{1 / 2} b^{s-r} L_{r}^{s-r}(-2 a b) \text { for } s \geqslant r,
$$

where $L_{k}^{\alpha}(x)$ is the generalized Laguerre polynomial. Depending on the energy levels $m, n$ that we are considering, the position of each Hermite polynomial should be modified to satisfy the requirement $s \geqslant r$, creating three different regimes $(N \leqslant M-1$, $N=M, N \geqslant M+1$ ) for each matrix product between operators. Taking the local limit, this scenario is greatly simplified, remaining only four contributions to the thermoelectric response function.

For the sake of clarity, consider as an example the electric current matrix element $J_{\mathbf{k} m s, \mathbf{k}+\mathbf{q} n s}^{x}$. After performing the spatial integration, the result is

$$
J_{\mathbf{k} m s, \mathbf{k}+\mathbf{q} n s}^{x}(\mathbf{q})=\frac{s v_{F} e}{(2 \pi)^{3 / 2}} \frac{e^{-\left(q_{x}^{2}+q_{y}^{2}\right) l_{B}^{2} / 4} e^{-i q_{y} l_{B}^{2}\left(k_{x}+q_{x} / 2\right)}}{\left[\alpha_{k_{z} m s}^{2}+1\right]^{\frac{1}{2}}\left[\alpha_{k_{z}+q_{z} n s}^{2}+1\right]^{\frac{1}{2}}} \Xi_{J x}(\mathbf{q}, m, n, s),
$$


where the function $\Xi_{J x}(\mathbf{q}, m, n, s)=\Xi_{1}^{\{i\}}+\Xi_{2}^{\{j\}}$ encodes all the information related with the different regimes between energy levels:

$$
\begin{aligned}
& \Xi_{1}^{\{1\}}(\mathbf{q}, m, n, s)=\alpha_{k_{z} m s} \sqrt{\frac{2^{N}(M-1) !}{2^{M-1} N !}}\left(\frac{-q_{x}-i q_{y}}{2} l_{B}\right)^{N-M+1} L_{M-1}^{N-M+1}\left(\frac{\mathbf{q}^{2} l_{B}^{2}}{2}\right) \quad(N \geqslant M-1), \\
& \Xi_{1}^{\{2\}}(\mathbf{q}, m, n, s)=\alpha_{k_{z} m s} \sqrt{\frac{2^{M-1} N !}{2^{N}(M-1) !}}\left(\frac{q_{x}-i q_{y}}{2} l_{B}\right)^{M-N-1} L_{N}^{M-N-1}\left(\frac{\mathbf{q}^{2} l_{B}^{2}}{2}\right) \quad(M \geqslant N+1), \\
& \Xi_{2}^{\{1\}}(\mathbf{q}, m, n, s)=\alpha_{k_{z}+q_{z} n s} \sqrt{\frac{2^{N-1} M !}{2^{M}(N-1) !}}\left(\frac{-q_{x}-i q_{y}}{2} l_{B}\right)^{N-M-1} L_{M}^{N-M-1}\left(\frac{\mathbf{q}^{2} l_{B}^{2}}{2}\right) \quad(N \geqslant M+1), \\
& \Xi_{2}^{\{2\}}(\mathbf{q}, m, n, s)=\alpha_{k_{z}+q_{z} n s} \sqrt{\frac{2^{M}(N-1) !}{2^{N-1} M !}}\left(\frac{q_{x}-i q_{y}}{2} l_{B}\right)^{M-N+1} L_{N-1}^{M-N+1}\left(\frac{\mathbf{q}^{2} l_{B}^{2}}{2}\right) \quad(M \geqslant N-1),
\end{aligned}
$$

where $\mathbf{q}^{2}=q_{x}^{2}+q_{y}^{2}$. The different terms in $\Xi$ are chosen depending on the scenario that we are studying. The matrix element of the electric current will be multiplied with the energy-momentum tensor terms (and their respective $\Xi$ functions). The elements of $\Xi_{J^{x}}$ and $\Xi_{T^{0 y}}$ to be multiplied will be selected according to the regime in consideration (detailed information about this type of operations can be found in Ref. [31]).

\section{Thermoelectric response function}

Computing the product of the electric current with the energy-momentum tensor (the three different parts being defined in the previous section), one gets the expression of the thermoelectric response function Eq. (22), which is made of two main terms:

$$
\begin{gathered}
\lim _{\mathbf{q} \rightarrow 0} \chi^{x y[1]}(\omega, \mathbf{q})=\frac{1}{4(2 \pi)^{2}} \sum_{\substack{m, n \\
N=M-1}} \frac{v_{F} e^{2} s^{2} B}{\hbar} \lim _{\eta \rightarrow 0^{+}} \int d \kappa_{z} \xi(\kappa, m, n, s, \omega, \eta)\left(\mathcal{E}_{\kappa_{z} m s}+\mathcal{E}_{\kappa_{z} n s}-2 \mu\right) \alpha_{\kappa_{z} m s}^{2}, \\
\lim _{\mathbf{q} \rightarrow 0} \chi^{x y[2]}(\omega, \mathbf{q})=\frac{1}{4(2 \pi)^{2}} \sum_{\substack{m, n \\
N=M-1}} \frac{-v_{F} e^{2} s B}{\hbar} \lim _{\eta \rightarrow 0^{+}} \int d \kappa_{z} \xi(\kappa, m, n, s, \omega, \eta)\left\{\alpha_{\kappa_{z} m s}^{2} \alpha_{\kappa_{z} n s} \sqrt{M-1}+\alpha_{\kappa_{z} m s} \sqrt{M}\right\},
\end{gathered}
$$

where the function $\xi(\kappa, m, n, s, \omega, \eta)$ is defined as:

$$
\xi(\kappa, m, n, s, \omega, \eta)=\frac{2\left[n_{\kappa m s}-n_{\kappa n s}\right]}{\left(\mathcal{E}_{\kappa_{z} m s}-\mathcal{E}_{\kappa_{z} n s}+i \frac{\hbar \eta}{v_{F} \sqrt{2 e B \hbar}}\right)\left(\mathcal{E}_{\kappa_{z} m s}-\mathcal{E}_{\kappa_{z} n s}+\frac{\hbar \omega}{v_{F} \sqrt{2 e B \hbar}}+i \frac{\hbar \eta}{v_{F} \sqrt{2 e B \hbar}}\right)} \frac{1}{\left[\alpha_{\kappa_{z} m s}^{2}+1\right]\left[\alpha_{\kappa_{z} n s}^{2}+1\right]} .
$$

Some relevant observations on Eqs. (B12) an (B13) are the following: as we see, the introduction of Dirac $\delta$ functions restricts the possible choices for the transitions between different Landau levels. They arise after computing the product of matrix elements [see the terms proportional to $\pm q_{x}-i q_{y}$ in Eqs. (B8)-(B11)], and the result is proportional to q to the $(N \pm M \pm 1)$ th power. In order to obtain a nonvanishing result in the local limit $(q \rightarrow 0)$, the exponents should be zero. The generalized Laguerre polynomials $L_{k}^{\alpha}(x)$, obtained from the product of Hermite polynomials, are equal to 1 when the local limit and the Dirac $\delta$ functions are evaluated. Concerning the dependence of the different factors on the chirality, no cancellations occur and both nodes contribute equally to the response function.

[1] R. A. Bertlmann, Anomalies in Quantum Field Theory (Clarendon, Oxford, 1996).

[2] B. R. Holstein, Anomalies for pedestrians, Am. J. Phys. 61, 142 (1993).

[3] D. E. Kharzeev, The chiral magnetic effect and anomalyinduced transport, Prog. Part. Nucl. Phys. 75, 133 (2014).
[4] K. Landsteiner, Anomalous transport of Weyl fermions in Weyl semimetals, Phys. Rev. B 89, 075124 (2014).

[5] H.-J. Kim, K.-S. Kim, J.-F. Wang, M. Sasaki, N. Satoh, A. Ohnishi, M. Kitaura, M. Yang, and L. Li, Dirac Versus Weyl Fermions in Topological Insulators: Adler-Bell-Jackiw 
Anomaly in Transport Phenomena, Phys. Rev. Lett. 111, 246603 (2013).

[6] J. Xiong et al., Evidence for the chiral anomaly in the Dirac semimetal $\mathrm{Na}_{3} \mathrm{Bi}$, Science 350, 413 (2015).

[7] G.-Z. Li et al., Giant negative magnetoresistance induced by the chiral anomaly in individual $\mathrm{Cd}_{3} \mathrm{As}_{2}$ nanowires, Nat. Commun. 6, 10137 (2015).

[8] C. Zhang et al., Signatures of the Adler Bell Jackiw chiral anomaly in a Weyl fermion semimetal, Nat. Commun. 7, 10735 (2016).

[9] J. Gooth et al., Experimental signatures of the mixed axial gravitational anomaly in the Weyl semimetal NbP, Nature 547, 23005 (2017).

[10] C. Schindler et al., Observation of an anomalous heat current in a Weyl fermion semimetal, arXiv:1810.02300.

[11] R. C. Tolman and P. Ehrenfest, Temperature equilibrium in a static gravitational field, Phys. Rev. 36, 1791 (1930).

[12] J. M. Luttinger, Theory of thermal transport coefficients, Phys. Rev. 135, A1505 (1964).

[13] T. Liang, J. Lin, Q. Gibson, T. Gao, M. Hirschberger, M. Liu, R. J. Cava, and N. P. Ong, Anomalous Nernst Effect in the Dirac Semimetal $\mathrm{Cd}_{3} \mathrm{As}_{2}$, Phys. Rev. Lett. 118, 136601 (2017).

[14] S. J. Watzman et al., Dirac dispersion generates unusually large Nernst effect in Weyl semimetals, Phys. Rev. B 97, 161404(R) (2018).

[15] M. N. Chernodub, A. Cortijo, and M. A. H. Vozmediano, Generation of a Nernst Current from the Conformal Anomaly in Dirac and Weyl Semimetals, Phys. Rev. Lett. 120, 206601 (2018).

[16] K. Behnia and H. Aubin, Nernst effect in metals and superconductors: A review of concepts and experiments, Rep. Prog. Phys. 79, 046502 (2016).

[17] R. Lundgren, P. Laurell, and G. A. Fiete, Thermoelectric properties of Weyl and Dirac semimetals, Phys. Rev. B 90, 165115 (2014).

[18] G. Sharma, P. Goswami, and S. Tewari, Nernst and magnetothermal conductivity in a lattice model of Weyl fermions, Phys. Rev. B 93, 035116 (2016).

[19] Y. Ferreiros, A. A. Zyuzin, and J. H. Bardarson, Anomalous Nernst and thermal hall effects in tilted Weyl semimetals, Phys. Rev. B 96, 115202 (2017).

[20] G. Sharma, C. Moore, S. Saha, and S. Tewari, Nernst effect in Dirac and inversion-asymmetric Weyl semimetals, Phys. Rev. B 96, 195119 (2017).

[21] E. V. Gorbar, V. A. Miransky, I. A. Shovkovy, and P. O. Sukhachov, Anomalous thermoelectric phenomena in lattice models of multi-Weyl semimetals, Phys. Rev. B 96, 155138 (2017).

[22] H. Yang et al., Giant anomalous nernst effect in the magnetic Weyl semimetal $\mathrm{Co}_{3} \mathrm{Sn}_{2} \mathrm{~S}$, arXiv:1811.03485.

[23] J. Gooth, G. Schierning, C. Felser, and K. Nielsch, Quantum materials for thermoelectricity, MRS Bull. 43, 187 (2018).

[24] B. Skinner and L. Fu, Large, nonsaturating thermopower in a quantizing magnetic field, Sci. Adv. 4, 2621 (2018).

[25] A. Sakai et al., Giant anomalous Nernst effect and quantumcritical scaling in a ferromagnetic semimetal, Nat. Phys. 14, 1119 (2018).

[26] K. Manna et al., From Colossal to Zero: Controlling the Anomalous Hall Effect in Magnetic Heusler Compounds via Berry Curvature Design, Phys. Rev. X 8, 041045 (2018).

[27] D. Xiao, Y. Yao, Z. Fang, and Q. Niu, Berry-Phase Effect in Anomalous Thermoelectric Transport, Phys. Rev. Lett. 97, 026603 (2006).

[28] N. P. Armitage, E. J. Mele, and A. Vishwanath, Weyl and Dirac semimetals in three-dimensional solids, Rev. Mod. Phys. 90, 015001 (2018).

[29] P. Hosur, S. A. Parameswaran, and A. Vishwanath, Charge Transport in Weyl Semimetals, Phys. Rev. Lett. 108, 046602 (2012).

[30] S. Jia, S. Xu, and M. Z. Hasan, Weyl semimetals, fermi arcs and chiral anomalies, Nat. Mater. 15, 1140 (2016).

[31] G. Giuliani and G. Vignale, Quantum Theory of the Electron Liquid (Cambridge University Press, Cambridge, 2005).

[32] N. R. Cooper, B. I. Halperin, and I. M. Ruzin, Thermoelectric response of an interacting two-dimensional electron gas in a quantizing magnetic field, Phys. Rev. B 55, 2344 (1997).

[33] T. Qin, Q. Niu, and J. Shi, Energy Magnetization and the Thermal Hall Effect, Phys. Rev. Lett. 107, 236601 (2011).

[34] P. Streda, Theory of quantised hall conductivity in two dimensions, J. Phys. C 15, L717 (1982).

[35] F. Han et al. Quantized plateau in the thermoelectric hall conductivity for Dirac electrons in the extreme quantum limit, arXiv:1904.02157.

[36] F. Han et al. Signature of the quantized thermoelectric hall effect in a topological Weyl semimetal, arXiv:1904.03179.

[37] V. Kozii, B. Skinner, and L. Fu, Thermoelectric hall conductivity and figure of merit in Dirac and Weyl materials, Phys. Rev. B 99, 155123 (2019).

[38] I. S. Gradshteyn and I. M. Ryzhik, Table of Integrals, Series, and Products, 7th ed. (Elsevier Academic Press, San Diego, CA, 2007). 